\title{
Exact Soliton Solutions for Generalized Equal Width Equation
}

\author{
N. Taghizadeh ${ }^{1}$, M. Mirzazadeh ${ }^{1}$, M. Akbari ${ }^{1}$ and M. Rahimian ${ }^{2}$ \\ ${ }^{1}$ Department of Mathematics, Faculty of Science, University of Guilan, Rasht, Iran \\ ${ }^{2}$ Department of Mathematics, Islamic Azad University, Masjed Soleiman Branch, Masjed Soleiman, Iran \\ Email: mirzazadehs2@guilan.ac.ir
}

Received: 4 Jan 2013; Revised: 6 Apr 2013; Accepted: 7 Apr 2013

Published online: 1 May. 2013

\begin{abstract}
The homogeneous balance method is used to construct exact travelling wave solutions of generalized equal width equation. Many exact travelling wave solutions of generalized equal width equation are successfully obtained, which contain soliton-like and periodic-like solutions. This method can be applied to nonintegrable equations as well as integrable ones.
\end{abstract}

Keywords: Homogeneous balance method; Generalized equal width equation; Riccati equation; Soliton-like solution; Periodic-like solution.

\section{Introduction}

The investigation of the traveling wave solutions of nonlinear partial differential equations plays an important role in the study of nonlinear physical phenomena. Nonlinear phenomena appear in a wide variety of scientific applications such as plasma physics, solid state physics, fluid dynamics. In recent years Wang [1, 2], Khalafallah [3, 4], used a useful homogeneous balance (HB) method for finding exact solutions of a given nonlinear partial differential equations. Fan [5] used HB method to search for Backlund transformation and similarity reduction of nonlinear partial differential equations. In this paper, we use the homogeneous balance method (HB) to solve the Riccati equation $\phi^{\prime}=a \phi^{2}+b \phi+c$ and the reduced nonlinear ordinary differential equation for generalized equal width (GEW) equation, respectively. It makes the HB method use more extensively.

\section{Generalized equal width equation}

For generalized equal width equation [7]

$$
u_{t}+\varepsilon u^{n} u_{x}-\delta u_{x x t}=0
$$

where $\varepsilon$ and $\delta$ are positive constants and $n$ is a positive integer.

Let us consider the travelling wave solutions

$$
u(x, t)=u(\xi), \quad \xi=k(x+\lambda t),
$$

where $k$ and $\lambda$ are constants.

Substituting (2) into (1), then (1) is reduced to the following nonlinear ordinary differential equation

$$
\lambda u^{\prime}+\varepsilon u^{n} u^{\prime}-\delta k^{2} \lambda u^{\prime \prime \prime}=0,
$$

where by integrating (3) and neglecting the constant of integration we obtain

$$
\lambda u+\frac{\varepsilon}{n+1} u^{n+1}-\delta k^{2} \lambda u^{\prime \prime}=0 .
$$

We now seek the solutions of Eq. (4) in the form 


$$
u=\left\{\sum_{i=0}^{m} a_{i} \phi^{i}\right\}^{\frac{1}{n}},
$$

where $a_{i}$ are constants to be determined later and $\phi$ satisfy the following Riccati equation

$$
\phi^{\prime}=a \phi^{2}+b \phi+c
$$

where $a, b$ and $c$ are constants.

Balancing $u^{n+1}$ with $u^{\prime \prime}$ in (4), we find

$$
(n+1) m=m+2
$$

so that

$$
m=\frac{2}{n} .
$$

To get a closed form solution, $m$ should be an integer. To achieve our goal, we use the transformation

$$
u(\xi)=v^{\frac{1}{n}}(\xi) \text {, }
$$

that will carry (4) into the nonlinear ordinary differential equation

$$
\lambda n^{2}(n+1) v^{2}+\varepsilon n^{2} v^{3}-\delta \lambda k^{2}\left(1-n^{2}\right)\left(v^{\prime}\right)^{2}-\delta \lambda k^{2} n(n+1) v v^{\prime \prime}=0 .
$$

Balancing $v^{3}$ with $v v^{\prime \prime}$ in (8) give

$$
3 m=2 m+2
$$

so that

$$
m=2 .
$$

The homogeneous balance method admits the use of the finite expansion in the form

$$
v=a_{0}+a_{1} \phi+a_{2} \phi^{2},
$$

where $a_{0}, a_{1}$ and $a_{2}$ are constants to be determined and $\phi$ satisfy Eq. (6).

Substituting Eqs. (9) and (6) into Eq. (8), and equating the coefficients of like powers of $\phi^{i}(i=0,1,2,3,4,5,6)$ to zero yields a set of algebraic system for $a_{0}, a_{1}, a_{2}, k$ and $\lambda$

$$
\begin{aligned}
& n^{2}(n+1) \lambda a_{0}^{2}+n^{2} \varepsilon a_{0}^{3}-n(n+1) \delta \lambda k^{2} a_{0}\left(a_{1} b c+2 c^{2} a_{2}\right)-\delta \lambda k^{2}\left(1-n^{2}\right) c^{2} a_{1}^{2}=0, \\
& -n(n+1) \delta \lambda k^{2} a_{0}\left(a_{1}\left(2 a c+b^{2}\right)+6 a_{2} b c\right)-n(n+1) \delta \lambda k^{2} a_{1}\left(a_{1} b c+2 a_{2} c^{2}\right)+2 n^{2}(n+1) \lambda a_{0} a_{1}+ \\
& 3 n^{2} \varepsilon a_{0}^{2} a_{1}-2 \delta \lambda k^{2}\left(1-n^{2}\right) a_{1} c\left(a_{1} b+2 a_{2} c\right)=0, \\
& n^{2}(n+1) \lambda\left(2 a_{0} a_{1}+a_{1}^{2}\right)-n(n+1) \delta \lambda k^{2} a_{0}\left(3 a b a_{1}+4 a_{2}\left(2 a c+b^{2}\right)\right)-n(n+1) \delta \lambda k^{2} a_{1} \\
& \left(a_{1}\left(2 a c+b^{2}\right)+6 a_{2} b c\right)-n(n+1) \delta \lambda k^{2} a_{2}\left(a_{1} b c+2 a_{2} c^{2}\right)-\delta \lambda k^{2}\left(1-n^{2}\right)\left(2 a_{1} c\left(a a_{1}+2 a_{2} b\right)\right. \\
& \left.+\left(a_{1} b+2 a_{2} c\right)^{2}\right)+n^{2} \varepsilon\left(a_{0}\left(2 a_{0} a_{2}+a_{1}^{2}\right)+2 a_{0} a_{1}^{2}+a_{2} a_{0}^{2}\right)=0, \\
& 2 n^{2}(n+1) \lambda a_{1} a_{2}-n(n+1) \delta \lambda k^{2} a_{0}\left(2 a_{1} a^{2}+10 a b a_{2}\right)-n(n+1) \delta \lambda k^{2} a_{1}\left(3 a b a_{1}+4 a_{2}\left(2 a c+b^{2}\right)\right) \\
& -n(n+1) \delta \lambda k^{2} a_{2}\left(a_{1}\left(2 a c+b^{2}\right)+6 b c a_{2}\right)-\delta \lambda k^{2}\left(1-n^{2}\right)\left(4 c a a_{1} a_{2}+2\left(a_{1} b+2 a_{2} c\right)\left(a a_{1}+2 a_{2} b\right)\right) \\
& +n^{2} \varepsilon\left(4 a_{0} a_{1} a_{2}+a_{1}\left(2 a_{0} a_{2}+a_{1}^{2}\right)\right)=0, \\
& -6 n(n+1) \delta \lambda k^{2} a^{2} a_{0} a_{2}-n(n+1) \delta \lambda k^{2} a_{1}\left(2 a^{2} a_{1}+10 a b a_{2}\right)-n(n+1) \delta \lambda k^{2} a_{2}\left(3 a b a_{1}\right. \\
& \left.+4 a_{2}\left(2 a c+b^{2}\right)\right)+n^{2}(n+1) \lambda a_{2}^{2}+n^{2} \varepsilon\left(a_{0} a_{2}^{2}+2 a_{1}^{2} a_{2}+a_{2}\left(2 a_{0} a_{2}+a_{1}^{2}\right)\right)-\delta \lambda k^{2}\left(1-n^{2}\right) \\
& \left(4\left(a_{1} b+2 a_{2} c\right) a a_{2}+\left(a a_{1}+2 a_{2} b\right)^{2}\right)=0,
\end{aligned}
$$




$$
\begin{aligned}
& -6 n(n+1) \delta \lambda k^{2} a_{1} a_{2} a^{2}-n(n+1) \delta \lambda k^{2} a_{2}\left(2 a^{2} a_{1}+10 a b a_{2}\right)-4 \delta \lambda k^{2}\left(1-n^{2}\right)\left(a a_{1}+2 a_{2} b\right) a a_{2} \\
& +3 n^{2} \varepsilon a_{1} a_{2}^{2}=0, \\
& -4 \delta \lambda k^{2}\left(1-n^{2}\right) a^{2} a_{2}^{2}+n^{2} \varepsilon a_{2}^{3}-6 n(n+1) \delta \lambda k^{2} a^{2} a_{2}^{2}=0 .
\end{aligned}
$$

For which, with the aid of Maple, we find

$$
\begin{gathered}
a_{0}=-\frac{2 a c \lambda(n+1)(n+2)}{\left(4 a c-b^{2}\right) \varepsilon}, a_{1}=-\frac{2 a b \lambda(n+1)(n+2)}{\left(4 a c-b^{2}\right) \varepsilon}, a_{2}=-\frac{2 a^{2} \lambda(n+1)(n+2)}{\left(4 a c-b^{2}\right) \varepsilon}, \\
k= \pm \frac{n}{\sqrt{\delta\left(b^{2}-4 a c\right)}} .
\end{gathered}
$$

It is to be noted that the Riccati equation (6) can be solved using the homogeneous balance method as follows

Case:I. Let $\phi=\sum_{i=0}^{m} b_{i} \tanh ^{i} \xi$. Balancing $\phi^{\prime}$ with $\phi^{2}$ leads to

$$
\phi=b_{0}+b_{1} \tanh \xi \text {. }
$$

Substituting Eq. (12) into Eq. (6), we obtain the following solution of Eq. (6)

$$
\phi=-\frac{1}{2 a}(b+2 \tanh \xi), \quad a c=\frac{b^{2}}{4}-1 .
$$

Substituting Eqs. (11) and (13) into (2), (7) and (9), we get the following traveling wave solutions of the generalized equal width (GEW) equation (1) :

$$
\begin{aligned}
& u(x, t)=\left\{\frac{(n+1)(n+2)}{\left(4 a c-b^{2}\right) \varepsilon}\left(\frac{b^{2}}{2}-2 a c-2 \tanh ^{2}\left[\frac{n}{\sqrt{\delta\left(b^{2}-4 a c\right)}}(x+\lambda t)\right]\right)\right\}^{\frac{1}{n}} \\
& =\left\{-\frac{(n+1)(n+2)}{2 \varepsilon} \sec h^{2}\left[\frac{n}{2 \sqrt{\delta}}(x+\lambda t)\right]\right\}^{\frac{1}{n}},
\end{aligned}
$$

where $a c=\frac{b^{2}}{4}-1$.

Similarly, let $\phi=\sum_{i=0}^{m} b_{i} \operatorname{coth}^{i} \xi$, then we obtain the following new traveling wave soliton solutions of the generalized equal width (GEW) equation (1):

$$
\begin{aligned}
& u(x, t)=\left\{\frac{(n+1)(n+2)}{\left(4 a c-b^{2}\right) \varepsilon}\left(\frac{b^{2}}{2}-2 a c-2 \operatorname{coth}^{2}\left[\frac{n}{\sqrt{\delta\left(b^{2}-4 a c\right)}}(x+\lambda t)\right]\right)\right\}^{\frac{1}{n}} \\
& =\left\{\frac{(n+1)(n+2)}{2 \varepsilon} \operatorname{csch}^{2}\left[\frac{n}{2 \sqrt{\delta}}(x+\lambda t)\right]\right\}^{\frac{1}{n}}
\end{aligned}
$$

where $a c=\frac{b^{2}}{4}-1$.

Case: II. From [6], when $a=1, \quad b=0$, the Riccati Eq. (6) has the following solutions

$$
\phi=-\sqrt{-c} \tanh (\sqrt{-c} \xi), \quad c<0,
$$




$$
\begin{array}{ll}
\phi=-\frac{1}{\xi}, & c=0, \\
\phi=\sqrt{c} \tan (\sqrt{c} \xi), & c>0 .
\end{array}
$$

From (7), (9), (11) and (16), we have the following new traveling wave solutions of generalized equal width (GEW) equation (1) which contain traveling wave solutions, periodic wave solutions and rational solutions as follows

When $c<0$, we have the following traveling wave (soliton-like) solutions of generalized equal width (GEW) equation (1) :

$$
u(x, t)=\left\{-\frac{2 \lambda(n+1)(n+2)}{\left(4 a c-b^{2}\right) \varepsilon}(a c-a b \sqrt{-c} \tanh (\sqrt{-c} \xi))-a^{2} c \tanh ^{2}(\sqrt{-c} \xi)\right\}^{\frac{1}{n}},
$$

where $\xi=\frac{n}{\sqrt{\delta\left(b^{2}-4 a c\right)}}(x+\lambda t)$.

When $c=0$, we have

$$
u(x, t)=\left\{-\frac{2 \lambda(n+1)(n+2)}{\left(4 a c-b^{2}\right) \varepsilon}\left(a c-\frac{a b}{\xi}-\frac{a^{2}}{\xi^{2}}\right)\right\}^{\frac{1}{n}}
$$

where $\xi=\frac{n}{\sqrt{\delta\left(b^{2}-4 a c\right)}}(x+\lambda t)$.

When $c>0$, we have the following traveling wave (periodic-like) solutions of generalized equal width (GEW) equation (1) :

$$
u(x, t)=\left\{-\frac{2 \lambda(n+1)(n+2)}{\left(4 a c-b^{2}\right) \varepsilon}(a c+a b \sqrt{c} \tan (\sqrt{c} \xi))+a^{2} c \tan ^{2}(\sqrt{c} \xi)\right\}^{\frac{1}{n}},
$$

where $\xi=\frac{n}{\sqrt{\delta\left(b^{2}-4 a c\right)}}(x+\lambda t)$.

Case: III. We suppose that the Riccati equation (6) has the following solutions of the form

$$
\phi=A_{0}+\sum_{i=1}^{m}\left(A_{i} f^{i}+B_{i} f^{i-1} g\right),
$$

with

which satisfy

$$
f=\frac{1}{\cosh \xi+r}, \quad g=\frac{\sinh \xi}{\cosh \xi+r},
$$

$$
\begin{gathered}
f^{\prime}(\xi)=-f(\xi) g(\xi), \quad g^{\prime}(\xi)=1-g^{2}(\xi)-r f(\xi), \\
g^{2}(\xi)=1-2 r f(\xi)+\left(r^{2}-1\right) f^{2}(\xi) .
\end{gathered}
$$

Balancing $\phi^{\prime}$ with $\phi^{2}$ leads to

$$
\phi=A_{0}+A_{1} f+B_{1} g \text {. }
$$

Substituting Eq. (21) into (6), collecting the coefficient of the same power $f^{i}(\xi) g^{i}(\xi)(i=0,1,2 ; j=0,1)$ and setting each of the obtained coefficients to zero yield the following set of algebra equations 


$$
\begin{aligned}
& a A_{0}^{2}+a B_{1}^{2}+b A_{0}+c=0, \\
& 2 a A_{0} A_{1}-2 a r B_{1}^{2}-r B_{1}+b A_{1}=0, \\
& a A_{1}^{2}+a\left(r^{2}-1\right) B_{1}^{2}+\left(r^{2}-1\right) B_{1}=0, \\
& 2 a A_{0} B_{1}+b B_{1}=0, \\
& 2 a A_{1} B_{1}+A_{1}=0,
\end{aligned}
$$

which have solutions

$$
A_{0}=-\frac{b}{2 a}, \quad A_{1}= \pm \sqrt{\frac{\left(r^{2}-1\right)}{4 a^{2}}}, \quad B_{1}=-\frac{1}{2 a}, \quad c=\frac{b^{2}-1}{4 a} .
$$

From Eqs. (21), (22), we obtain

$$
\phi=-\frac{1}{2 a}\left(b+\frac{\sinh \xi \mp \sqrt{\left(r^{2}-1\right)}}{\cosh \xi+r}\right) .
$$

From Eqs. (7), (9), (11) and (23), we obtain the new solutions of generalized equal width (GEW) equation (1):

$$
u(x, t)=\left\{\frac{\lambda(n+1)(n+2)}{\left(4 a c-b^{2}\right) \varepsilon}\left(\frac{b^{2}}{2}-2 a c-\frac{1}{2}\left(\frac{\sinh \xi \mp \sqrt{\left(r^{2}-1\right)}}{\cosh \xi+r}\right)^{2}\right)\right\}^{\frac{1}{n}},
$$

where $\xi=\frac{n}{\sqrt{\delta\left(b^{2}-4 a c\right)}}(x+\lambda t)$.

Case: IV. We take $\phi$ in the Riccati equation (6) being of the form

$$
\phi=e^{p_{1} \xi} \rho(z)+p_{4}(\xi)
$$

where

$$
z=e^{p_{2} \xi}+p_{3},
$$

where $p_{1}, p_{2}$ and $p_{3}$ are constants to be determined.

Substituting (25) into (6) we find that when $c=\frac{-p_{1}^{2}+b^{2}}{4 a}$, we have

$$
\phi=-\frac{p_{1} e^{p_{1} \xi}}{a\left(e^{p_{1} \xi}+p_{3}\right)}+\frac{p_{1}-b}{2 a} .
$$

If $p_{3}=1$ in (26), we have

$$
\phi=-\frac{p_{1}}{2 a} \tanh \left(\frac{1}{2} p_{1} \xi\right)-\frac{b}{2 a} .
$$

If $p_{3}=-1$ in (26), we have

$$
\phi=-\frac{p_{1}}{2 a} \operatorname{coth}\left(\frac{1}{2} p_{1} \xi\right)-\frac{b}{2 a} \text {. }
$$

From (7), (9), (11) and (26), we obtain the following new traveling wave solutions of generalized equal width (GEW) equation (1):

$$
u(x, t)=\left\{-\frac{2 \lambda(n+1)(n+2)}{\left(4 a c-b^{2}\right) \varepsilon}\left(a c+\left(\frac{p_{1}+b}{2}-\frac{\left.p_{1} e^{p_{1}\left(\frac{n}{\sqrt{\delta\left(b^{2}-4 a c\right)}}(x+\lambda t)\right.}\right)}{\left.e^{p_{1}\left(\frac{n}{\sqrt{\delta\left(b^{2}-4 a c\right)}}(x+\lambda t)\right.}\right)}+p_{3}\right)\left(\frac{p_{1}-b}{2}-\frac{\left.p_{1} e^{p_{1}\left(\frac{n}{\sqrt{\delta\left(b^{2}-4 a c\right)}}(x+\lambda t)\right.}\right)}{\left.e^{p_{1}\left(\frac{n}{\sqrt{\delta\left(b^{2}-4 a c\right.}}(x+\lambda t)\right.}\right)}+p_{3}\right)\right)\right\}^{\frac{1}{n}} .
$$

When $p_{3}=1$, we have the following traveling wave (soliton-like) solutions of generalized equal width (GEW) equation (1) : 


$$
u(x, t)=\left\{\frac{(n+1)(n+2)}{\left(4 a c-b^{2}\right) \varepsilon}\left(\frac{b^{2}}{2}-2 a c-\frac{p_{1}^{2}}{2} \tanh ^{2}\left(\frac{p_{1}}{2}\left(\frac{n}{\sqrt{\delta\left(b^{2}-4 a c\right)}}(x+\lambda t)\right)\right)\right)\right\}^{\frac{1}{n}} .
$$

When $p_{3}=-1$, we have the following traveling wave (periodic-like) solutions of equal width wave equation (1) :

$$
u(x, t)=\left\{\frac{(n+1)(n+2)}{\left(4 a c-b^{2}\right) \varepsilon}\left(\frac{b^{2}}{2}-2 a c-\frac{p_{1}^{2}}{2} \operatorname{coth}^{2}\left(\frac{p_{1}}{2}\left(\frac{n}{\sqrt{\delta\left(b^{2}-4 a c\right)}}(x+\lambda t)\right)\right)\right)\right\}^{\frac{1}{n}} .
$$

Case: V. We suppose that the Riccati equation (6) have the following solutions of the form:

$$
\phi=A_{0}+\sum_{i=1}^{m} \sinh ^{i-1}\left(A_{i} \sinh w+B_{i} \cosh w\right),
$$

where $\frac{d w}{d \xi}=\sinh w$ or $\frac{d w}{d \xi}=\cosh w$. It is easy to find that $m=1$ by balancing $\phi^{\prime}$ and $\phi^{2}$.

So we choose

$$
\phi=A_{0}+A_{1} \sinh w+B_{1} \cosh w,
$$

when $\frac{d w}{d \xi}=\sinh w$, we substitute (33) and $\frac{d w}{d \xi}=\sinh w$, into (6) and set the coefficients of $\sinh ^{i} w \cosh ^{i} w(i=0,1,2 ; j=0,1)$ to zero. A set of algebraic equations is obtained as follows:

$$
\begin{aligned}
& a A_{0}^{2}+a B_{1}^{2}+b A_{0}+c=0, \\
& 2 a A_{0} A_{1}+b A_{1}=0, \\
& a A_{1}^{2}+a B_{1}^{2}-B_{1}=0, \\
& 2 a A_{0} B_{1}+b B_{1}=0, \\
& 2 a A_{1} B_{1}+A_{1}=0,
\end{aligned}
$$

for which, we have the following solutions:

$$
A_{0}=-\frac{b}{2 a}, \quad A_{1}=0, \quad B_{1}=\frac{1}{a},
$$

where $c=\frac{b^{2}-4}{4 a}$, and

$$
A_{0}=-\frac{b}{2 a}, \quad A_{1}= \pm \sqrt{\frac{1}{2 a}}, \quad B_{1}=\frac{1}{2 a},
$$

where $c=\frac{b^{2}-1}{4 a}$.

To $\frac{d w}{d \xi}=\sinh w$, we have

$$
\sinh w=-\operatorname{csch} \xi, \quad \cosh w=-\operatorname{coth} \xi .
$$

From (34) - (36), we obtain

$$
\phi=-\frac{b+2 \operatorname{coth} \xi}{2 a},
$$

where $c=\frac{b^{2}-4}{4 a}$, and 


$$
\phi=-\frac{b \pm \csc h \xi+\operatorname{coth} \xi}{2 a},
$$

where $c=\frac{b^{2}-1}{4 a}$.

From Eqs. (7), (9), (11), (37) and (38) we get the following traveling wave solutions of the generalized equal width (GEW) equation (1):

$$
u(x, t)=\left\{\frac{(n+1)(n+2)}{2 \varepsilon} \operatorname{csch}^{2}\left(\frac{n}{2 \sqrt{\delta}}(x+\lambda t)\right)\right\}^{\frac{1}{n}}
$$

where $a c=\frac{b^{2}}{4}-1$. and

$$
u(x, t)=\left\{\frac{(n+1)(n+2)}{\left(4 a c-b^{2}\right) \varepsilon}\left(a c-\frac{b}{2}(b \pm \operatorname{csch} \xi+\operatorname{coth} \xi)+\frac{1}{4}(b \pm \operatorname{csch} \xi+\operatorname{coth} \xi)^{2}\right)\right\}^{\frac{1}{n}}
$$

where $c=\frac{b^{2}-1}{4 a}$ and $\xi=\frac{n}{\sqrt{\delta\left(b^{2}-4 a c\right)}}(x+\lambda t)$.

Similarly, when $\frac{d w}{d \xi}=\cosh w$, we obtain the following traveling wave (periodic-like) solutions of the generalized equal width (GEW) equation (1) :

$$
u(x, t)=\left\{\frac{(n+1)(n+2)}{2 \varepsilon} \csc ^{2}\left(\frac{n}{2 \sqrt{\delta}}(x+\lambda t)\right)\right\}^{\frac{1}{n}},
$$

where $a c=\frac{b^{2}}{4}-1$. and

$$
\begin{aligned}
& u(x, t)=\left\{\frac{(n+1)(n+2)}{\left(4 a c-b^{2}\right) \varepsilon}\left(a c-\frac{b}{2}(b \pm \csc \xi+\cot \xi)+\frac{1}{4}(b \pm \csc \xi+\cot \xi)^{2}\right)\right\}^{\frac{1}{n}}, \\
& \text { where } \quad c=\frac{b^{2}-1}{4 a} .
\end{aligned}
$$

In summary we have used the extended homogeneous balance method to obtain many traveling wave solutions of the generalized equal width (GEW) equation.

We now summarize the key steps as follows:

Step 1: For a given nonlinear evolution equation

$$
F\left(u, u_{t}, u_{x}, u_{x t}, u_{t t}, \ldots\right)=0 \text {, }
$$

we consider its travelling wave solutions $u(x, t)=u(\xi), \quad \xi=k(x+l t)+d \quad$ then Eq. (43) is reduced to an nonlinear ordinary differential equation

$$
Q\left(u, u^{\prime}, u^{\prime \prime}, u^{\prime \prime \prime}, \ldots\right)=0 \text {, }
$$

where a prime denotes $\frac{d}{d \xi}$.

Step 2: For a given ansatz equation (for example, the ansatz equation is $\phi^{\prime}=a \phi^{2}+b \phi+c$ in this paper), the form of $u$ is decided and the homogeneous balance method is used on Eq. (44) to find the coefficients of $u$.

Step 3: The homogeneous balance method is used to solve the ansatz equation.

Step 4: Finally, the travelling wave solutions of Eq. (43) are obtained by combining steps 2 and 3. 


\section{Acknowledgement}

The authors are very grateful to the referees for their detailed comments and kind help.

\section{References}

[1] M. L. Wang, Solitrary wave solution for variant Boussinesq equation, Phys.Lett. A, 199, 169-172 (1995).

[2] M. L. Wang, Applicatian of homogeneous balance method to exact solutions of nonlinear equation in mathematical physics, Phys. Lett. A, 216, 67-75 (1996).

[3] M. Khalfallah, New exact traveling wave solutions of the $(3+1)$ dimensional Kadomtsev-Petviashvili $(K P)$ equation, Commun. Nonlinear Sci. Numer. Simul., 14, 1169-1175 (2009).

[4] M. Khalfallah, Exact traveling wave solutions of the Boussinesq-Burger equation, Math. Comput. Model., 49, 666-671 (2009).

[5] E. G. Fan, Two new applications of the homogeneous balance method, Phys.Lett. A, 265, 353-357 (2000).

[6] X. Q. Zhao, D. B. Tang, A new note on a homogeneous balance method, Phys. Lett. A, 297, 59-67 (2002).

[7] D. J. Evans and K. R. Raslan, Solitary waves for the generalized equal width (GEW) equation, International Journal of Computer Mathematics, 82(4), 445-455 (2005). 